STRUCTURAL BIOLOGY COMMUNICATIONS

ISSN 2053-230X

Received 22 May 2021

Accepted 5 August 2021

Edited by M. A. Hough, University of Essex, United Kingdom

Keywords: Yersinia pestis; YfeA site 2; substrate-binding proteins; inter-protein metal coordination; crystallography; zinc; manganese; transition-metal homeostasis; plague.

PDB references: YfeA oligomer crystal 1, form 1,7me1; crystal 2, form 2, $7 \mathrm{me} 2$; crystal 3, form 2, $7 \mathrm{me} 3$

Supporting information: this article has supporting information at journals.iucr.org/f

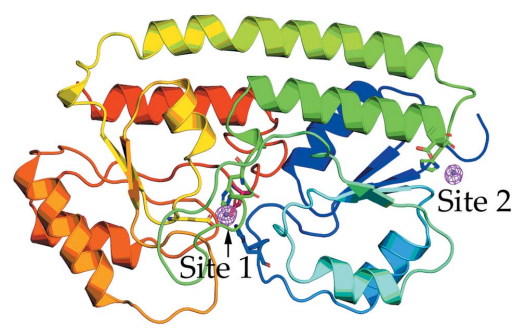
OPEN ○ ACCESS

\section{Site 2 of the Yersinia pestis substrate-binding protein YfeA is a dynamic surface metal-binding site}

\author{
Christopher D. Radka ${ }^{a}$ and Stephen G. Aller ${ }^{b *}$ \\ a'Department of Infectious Diseases, St Jude Children's Research Hospital, Memphis, TN 38105, USA, and ${ }^{\mathbf{b}}$ Department of \\ Pharmacology and Toxicology, University of Alabama at Birmingham, Birmingham, AL 35294, USA. *Correspondence \\ e-mail: sgaller@uab.edu
}

The substrate-binding protein YfeA (also known as YPO2439 or y1897) is a polyspecific metal-binding protein that is crucial for nutrient acquisition and virulence in Yersinia pestis, the causative microbe of plague. YfeA folds into a monomeric c-clamp like other substrate-binding proteins and has two metalbinding sites (sites 1 and 2). Site 2 is a bidentate surface site capable of binding $\mathrm{Zn}$ and $\mathrm{Mn}$ atoms and is a unique feature of YfeA. Occasionally, the site 2 residues of two YfeA molecules will cooperate with the histidine tag of a third YfeA molecule in coordinating the same metal and lead to metal-dependent crystallographic packing. Here, three crystal structures of YfeA are presented at $1.85,2.05$ and $2.25 \AA$ resolution. A comparison of the structures reveals that the metal can be displaced at five different locations ranging from $\sim 4$ to $\sim 16 \AA$ away from the canonical site 2 . These observations reveal different configurations of site 2 that enable cooperative metal binding and demonstrate how site 2 is dynamic and freely available for inter-protein metal coordination.

\section{Introduction}

Plague is an infection by Yersinia pestis that has caused several pandemics and reached all corners of the globe (Vogler et al., 2013). Recent outbreaks in Africa (Respicio-Kingry et al., 2016; Abedi et al., 2018; Andrianaivoarimanana et al., 2019; Randremanana et al., 2019) and China (Shi et al., 2018) show that plague remains a threat to public health. Transition-metal acquisition is a key process in plague pathogenesis. Disruption of the manganese and iron transporter Yfe renders $Y$. pestis avirulent by intravenous infection or severely compromises virulence on subcutaneous infection (Bearden \& Perry, 1999). Yfe is an ATP-binding cassette transporter made up of the substrate-binding protein (SBP) YfeA, the ATP-binding protein $\mathrm{YfeB}$ and the membrane components $\mathrm{YfeCDE}$ (Bearden et al., 1998).

The crystal structure of YfeA shows the prototypical SBP c-clamp fold with a primary substrate-binding site located in the c-clamp arch (site 1; Scheepers et al., 2016). SBPs are classified into clusters according to their three-dimensional structures and the clusters correlate with the substrate specificity (Scheepers et al., 2016). YfeA is a cluster A-I SBP that binds metal atoms such that site 1 binds $\mathrm{Zn}, \mathrm{Fe}$ and $\mathrm{Mn}$ atoms and a secondary substrate-binding site located on the surface (site 2) binds $\mathrm{Zn}$ and $\mathrm{Mn}$ atoms (Fig. 1a; Radka et al., 2017). Both sites 1 and 2 are tetracoordinate, where four amino acids (His76/His141/Glu207/Asp282) coordinate the metal in site 1 and two amino acids (Glu162/His163) and two water molecules coordinate the metal in site 2 (Radka et al., 2017). Multiple surface ancillary binding sites can be reversibly occupied by soaking YfeA crystals in zinc or back-soaking in 
metal-free mother liquor, but site 2 requires zinc or manganese to be present during crystallization and is not demetalated by back-soaking (Radka et al., 2017). Furthermore, metal occupancy at site 2 influences which grouping of surface ancillary sites bind metal (Radka et al., 2017). There is no clear path to shuttle substrate across the $>26 \AA$ distance between sites 1 and 2, and methods to generate apo YfeA (Radka et al., 2018) and reconstitute site 1 metal-bound holo YfeA (Radka et al., 2019) showed that site 2 does not play an apparent role in loading metal into site 1.

Here, structures in which metals are inter-coordinated by multiple YfeA molecules are reported at 1.85, 2.05 and $2.25 \AA$ resolution. The crystal structures show how a metal can bounce around site 2 and trigger distinct assemblies of protein-protein interactions. The metal recruits site 2 residues, other surface residues and $\mathrm{His}_{10}$ tag residues from multiple protomers to collaborate in metal coordination and form crystal contacts in the lattice. These modes of engaging YfeA at site 2 presents the intriguing possibility that site 2 may participate in protein-protein interactions with other metalbinding proteins.

\section{Experimental procedures}

\subsection{Production and purification of $\mathrm{Yfe} A$}

Recombinant YfeA-His ${ }_{10}$ was overexpressed in Escherichia coli strain BL21(DE3) pLysS Singles (Novagen; catalog No. 70236) using the vector pET-22b and purified as described previously (Radka et al., 2017). Briefly, YfeA-His ${ }_{10}$ was loaded onto a HisTrap HP column (GE Healthcare Life Sciences; catalog No. 17-5248-02), washed and eluted using a linear imidazole gradient from 0.02 to $1.0 \mathrm{M}$ imidazole. Fractions containing the eluent were pooled and loaded onto a HiTrap Q HP column (GE Healthcare Life Sciences; catalog No. 171154-01), washed and eluted using a linear $\mathrm{NaCl}$ gradient from 0.0 to $1.0 \mathrm{M} \mathrm{NaCl}$. Fractions containing the eluent were pooled and further purified by gel filtration on a HiLoad 26/600 Superdex 200 pg column (GE Healthcare Life Sciences; catalog No. 28-9893-36). The final purified protein was concentrated to $18 \pm 5 \mathrm{mg} \mathrm{ml}^{-1}$ in $20 \mathrm{~m} M$ bis-Tris propane $\mathrm{pH}$ $6.3,50 \mathrm{mM} \mathrm{NaCl}, 0.05 \%(w / v) \mathrm{NaN}_{3}$ for crystallization.

\subsection{Analysis of oligomeric state}

To determine whether $\mathrm{MnCl}_{2}$ triggered YfeA oligomerization, $5 \mathrm{mg} \mathrm{ml}^{-1}$ YfeA was incubated with and without $10 \mathrm{mM}$ $\mathrm{MnCl}_{2}$ in $20 \mathrm{~m} M$ bis-Tris propane $\mathrm{pH} 6.3,50 \mathrm{~m} M \mathrm{NaCl}$, $0.05 \%(w / v) \mathrm{NaN}_{3}$ for $2.5 \mathrm{~h}$ at room temperature. The mixture was analyzed by gel filtration using a Superdex 200 10/300 GL column (Cytiva Life Sciences; catalog No. 17517501).

\subsection{Crystallization, data collection and structure determination}

Crystals of YfeA-His ${ }_{10}$ were grown by the hanging-drop vapor-diffusion method at $20^{\circ} \mathrm{C}$ using a $1: 1$ ratio of $32 \%(w / v)$ PEG 4000, $20 \mathrm{~m} M$ bis-Tris propane $\mathrm{pH} 6.3,50 \mathrm{~m} M \mathrm{NaCl}$, $0.05 \%(w / v) \mathrm{NaN}_{3}, 10 \mathrm{~m} M \mathrm{MnCl}_{2}$ and $18 \pm 5 \mathrm{mg} \mathrm{ml}^{-1} \mathrm{YfeA}-$
His $_{10}$. X-ray diffraction data were collected at the X-ray absorption-edge energy ( $K$ edge) for zinc or iron as described previously (Radka et al., 2017). These energies were determined at the beamline for each data-collection session. Data collected near the $\mathrm{Fe} K$ edge can visualize both iron and manganese because the Fe $K$-edge energy is greater than the Mn $K$-edge energy. Collecting data at the lower Mn $K$-edge energy would visualize manganese but not iron or zinc. Iron and manganese are only weakly detected at the $\mathrm{Zn} K$ edge. The data were merged and scaled using $H K L-2000$ version 715 (Otwinowski \& Minor, 1997). The structure was solved by molecular replacement using Phaser (Zwart et al., 2008) as implemented in Phenix (Liebschner et al., 2019) and monomeric YfeA (PDB entry 5uxs) was used as a search model (Radka et al., 2017). Additional model building and structural refinement were performed using Coot (Emsley et al., 2010) and Phenix (Liebschner et al., 2019). The figures were generated using PyMOL (DeLano, 2002). Statistics for the X-ray data processing and model refinement are listed in Table $1 . \mathrm{C}^{\alpha}$ r.m.s.d. calculations were performed using secondary-structure matching (SSM) Superpose in Coot (Emsley et al., 2010).

\section{Results}

Approximately $5 \%$ of the YfeA crystals grown in the presence of $\mathrm{MnCl}_{2}$ belonged to an orthorhombic space group with unitcell dimensions $a=67.3 \pm 0.2, b=76.4 \pm 0.6, c=108.1 \pm 1.9 \AA$ (Table 1) and two YfeA protomers in the asymmetric unit (Fig. 1b), whereas generally the YfeA crystals had one YfeA molecule in the asymmetric unit and belonged to an orthorhombic space group with unit-cell dimensions $a=41, b=52$, $c=113 \AA$ or $a=62, b=66, c=66 \AA$ (Radka et al., 2017). Inspection of the arrangement of the YfeA protomers shows crystallographic packing such that site 2 of one protomer is in close proximity to site 2 from a second protomer and the $\mathrm{His}_{10}$ tag from a third protomer (Fig. 1c). Structural alignment of each YfeA protomer from PDB entries 5uyg and 5uxs (monomeric YfeA with and without metal bound in site 2, respectively) yielded an r.m.s.d. of $0.54 \pm 0.09 \AA$ for $269-272$ pairs of matched $\mathrm{C}^{\alpha}$ atoms, indicating that the YfeA protomers here are highly similar to YfeA monomers. The r.m.s.d. among the present YfeA protomers is $0.47 \pm 0.16 \AA$ for 271 281 pairs of matched $\mathrm{C}^{\alpha}$ atoms, indicating that the YfeA protomers are highly similar to one another. Some protomer pairs had more matched $\mathrm{C}^{\alpha}$ atoms than others due to the resolution of additional residues in the carboxy-terminal $\mathrm{His}_{10}$ tag in some protomers. YfeA can also bind ancillary metals at additional surface sites (Fig. 1d). These metals are coordinated by multiple protomers and enable crystal contacts in the lattice, but have incomplete coordination spheres due to missing solvent molecules or other nonprotein ligands (for example buffer molecules; Laitaoja et al., 2013).

The site 2 substrate will be simply referred to as 'the metal' because site 2 binds zinc and manganese (Radka et al., 2017), as also shown by the anomalous difference density from the diffraction data collected at the $K$ edges (Section 2), and the 
Table 1

Data-collection and refinement statistics.

Values in parentheses are for the highest resolution shell.

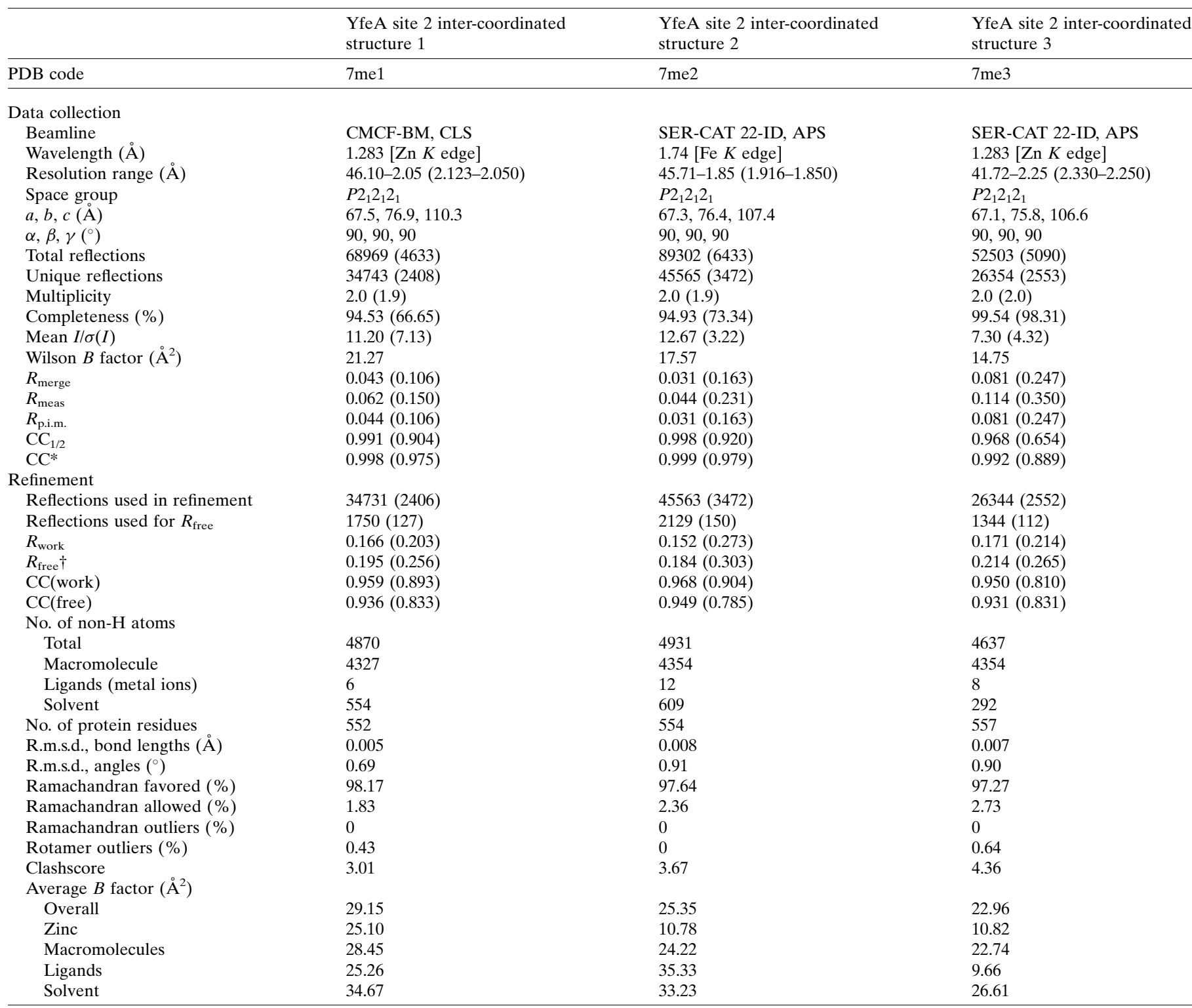

$\dagger 5 \%$ of the data were reserved for calculating the $R_{\text {free }}$ statistic.

coordination geometries in the crystal structures can apply to both.

In YfeA site 2 inter-coordinated structure 1, the metal is coordinated by the backbone carbonyl of site 2 residue His 163 of one protomer, the side chain of surface residue Asp114 of a second protomer, $\mathrm{His}_{10}$-tag residues His314 and His316 of a third protomer and a water molecule (Fig. 2a). Three histidine residues of the $\mathrm{His}_{10}$ tag are resolved in one protomer and no histidine residues are resolved in the other protomer in the asymmetric unit. The arrangement of ligands results in square bipyramidal coordination geometry, where the His314 ligand is at the apex of the pyramid and the other four ligands form the square base. Square pyramidal coordination geometry is commonly observed in protein-manganese (Udayalaxmi et al., 2020) and protein-zinc (Laitaoja et al., 2013) complexes.
In YfeA site 2 inter-coordinated structure 2, the metal is coordinated by the side chain of site 2 residue His 163 of one protomer, the side chain of site 2 residue Glu162 of a second protomer and $\mathrm{His}_{10}$-tag residues His314 and His316 of a third protomer (Fig. $2 b$ ). Therefore, two site 2 motifs participate in metal coordination. Five histidine residues of the $\mathrm{His}_{10}$ tag are resolved in one protomer and no histidine residues are resolved in the other protomer in the asymmetric unit. The four ligands form bond angles of $\sim 110-115^{\circ}$ in this arrangement, which is consistent with tetrahedral coordination geometry. Tetrahedral coordination geometry is widespread in protein-transition metal complexes and is used at site 1 to coordinate zinc, manganese and iron (Radka et al., 2017). Data for structure 2 were collected at the Fe $K$ edge specifically because (i) Yfe is required for iron transport and full virulence 
of plague infection (Bearden \& Perry (1999), (ii) the structure reveals unique metal binding that is not present in the other two structures and (iii) the X-ray energy confirms the presence of iron or manganese but cannot detect zinc anomalous scattering.

In YfeA site 2 inter-coordinated structure 3, the metal is coordinated by the same ligands as in structure 2 to achieve tetrahedral coordination geometry (Fig. 2c). Similarly, five histidine residues of the $\mathrm{His}_{10}$ tag are resolved in one protomer and no histidine residues are resolved in the other protomer in the asymmetric unit. There are no ancillary metals present in the structure.

Soluble YfeA was incubated in crystallization buffer with and without $10 \mathrm{mM} \mathrm{MnCl}_{2}$ for $2.5 \mathrm{~h}$ and the mixtures were seperated by gel filtration to determine whether $\mathrm{MnCl}_{2}$ triggered YfeA oligomerization in solution. The elution position under both conditions is consistent with monomeric YfeA, indicating that the perceived oligomerization is a property of crystallization (Fig. 3). The crystal-packing contacts are likely to stabilize the arrangement of protein molecules in the crystal

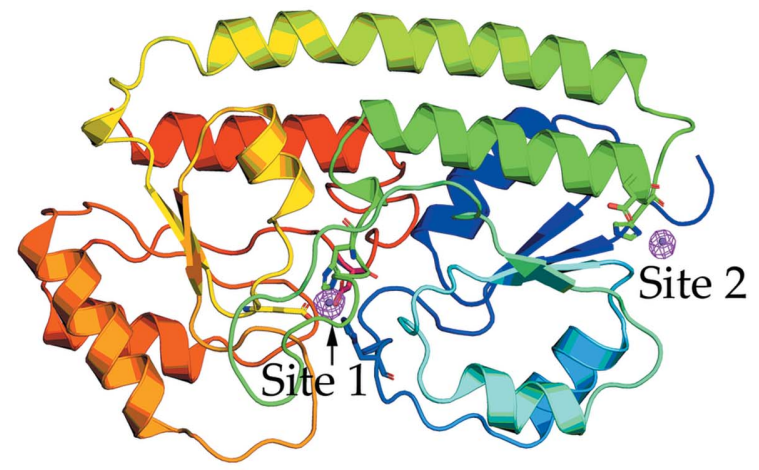

(a)

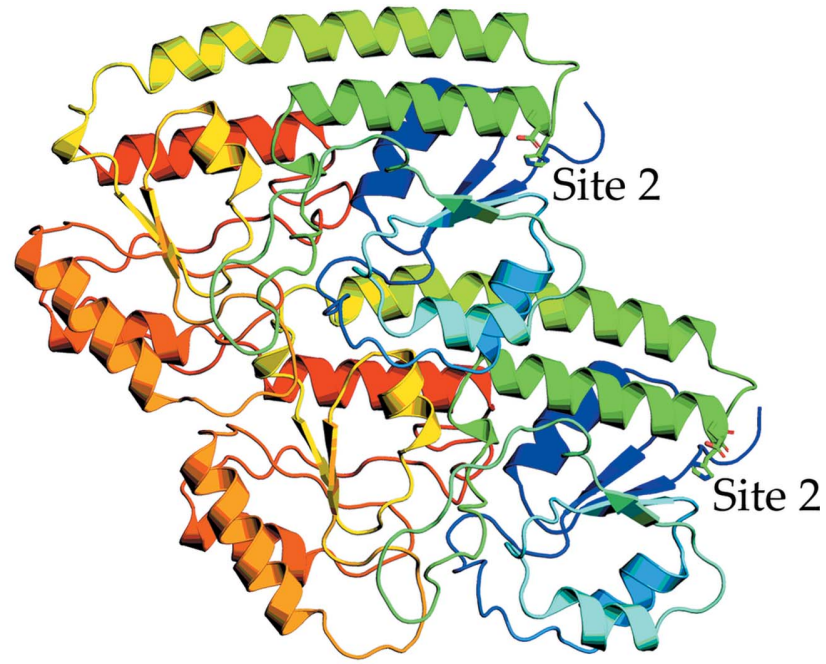

(b)

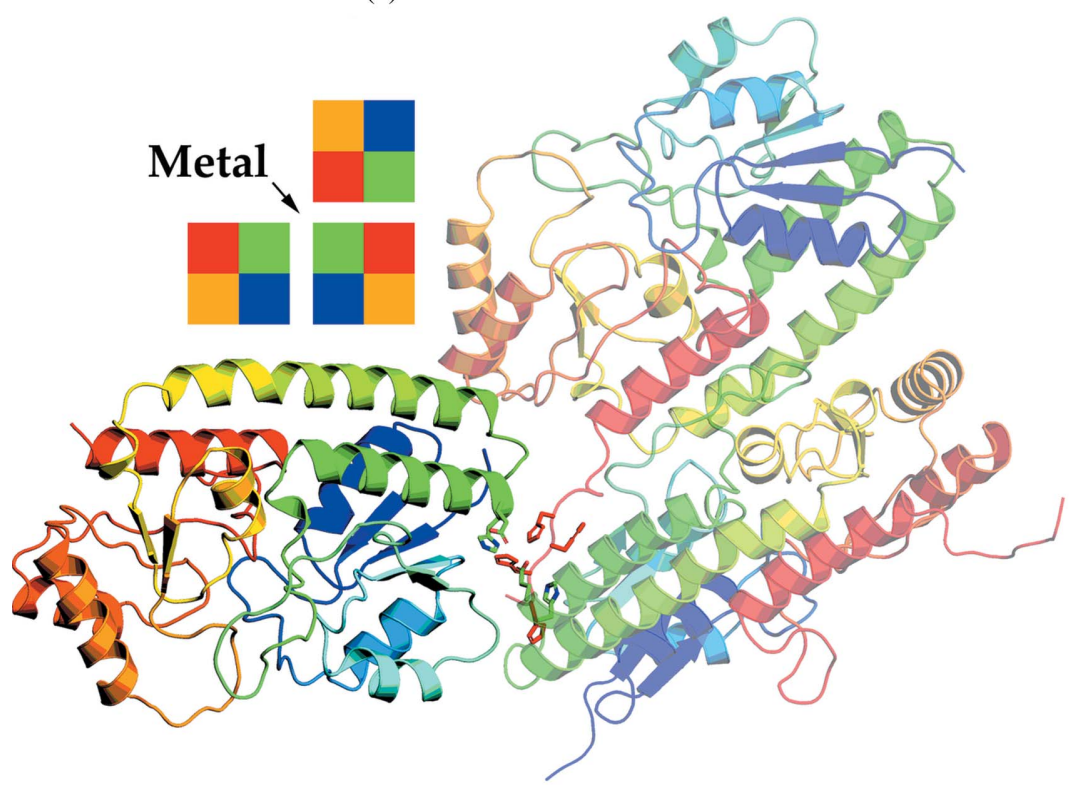

(c)

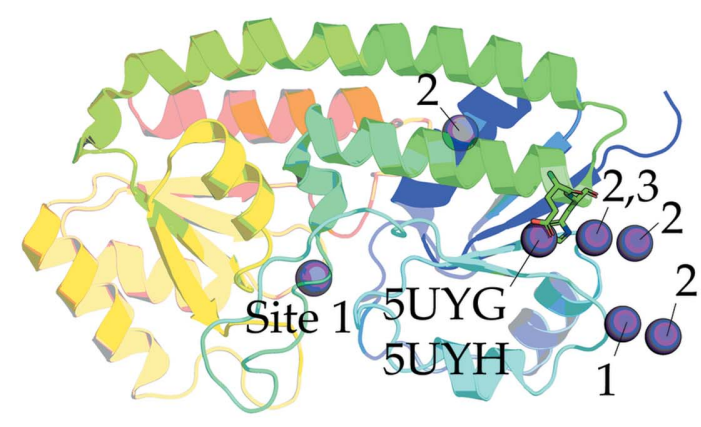

(d)

Figure 1

Arrangement of YfeA protomers and crystallographic packing. YfeA protomers are colored in a spectrum from blue (amino-terminus) to red (carboxyterminus) and metals are shown as spheres. (a) Site 1 is a buried metal-binding site with four amino-acid ligands and site 2 is a surface site with two amino-acid ligands. Anomalous difference electron density contoured at $4 \sigma$ (magenta mesh) shows the location of each metal. (b) Two YfeA protomers are present in the asymmetric unit of each crystal and the site 2 motifs are distant from each other. (c) Each protomer interacts with two symmetryrelated protomers (shown as transparent molecules) to form a crystallographic complex. In the arrangement shown, two site 2 motifs (green) flank residues from a $\mathrm{His}_{10}$ tag (red). The protomers in the complex are related by rotation and reflection symmetry along the screw axis. Blocks colored according to the YfeA sequence show the symmetry relationship between protomers and how the metal is wedged between protomers. $(d)$ Overview of the positional distribution of metals in the YfeA crystal packing. A metal is present in site 1 in all structures. PDB entries 5uyg and 5uyh show the canonical site 2 metal site. Metals present in YfeA site 2 inter-coordinated structure 1, structure 2 and structure 3 are denoted 1, 2 and 3, respectively. A common metal is present in structures 2 and 3. 
lattice and the $\mathrm{MnCl}_{2}$ in the mother liquor enabled YfeA nucleation around site 2 in a metal-binding-dependent manner.

\section{Discussion}

The bacterial ferritin FtnA contains a glutamate-histidine Featom binding site that is the final observable stop $\left(\mathrm{Fe}_{\mathrm{C}}\right)$ in an internal translocation relay before iron is incorporated into a growing mineral for nutrient storage (Yao et al., 2011). Proton transfer within the interior cavity is proposed as a key process to promote complementary ionization states of the binding residues with oxidation states of the iron for translocation relay (Yao et al., 2011). Since the YfeA Glu-His site 2 is on the surface, the modulation of charge potential for substrate

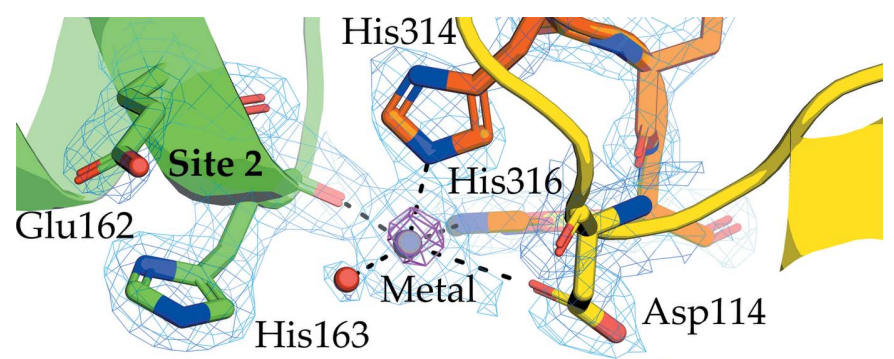

(a)

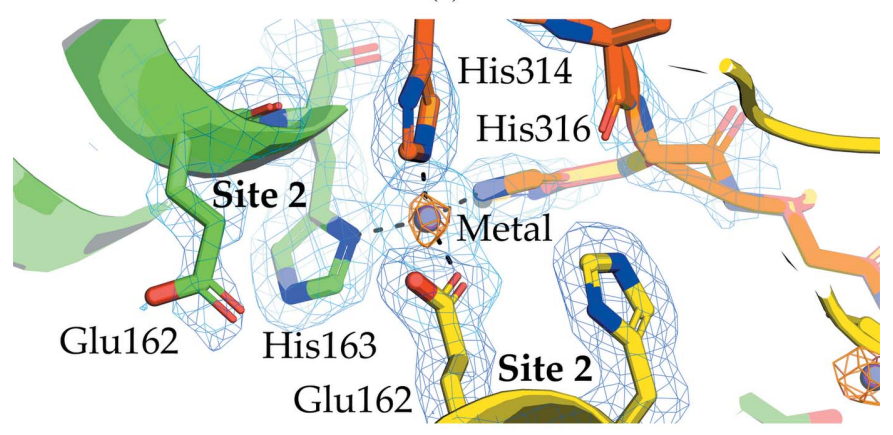

(b)

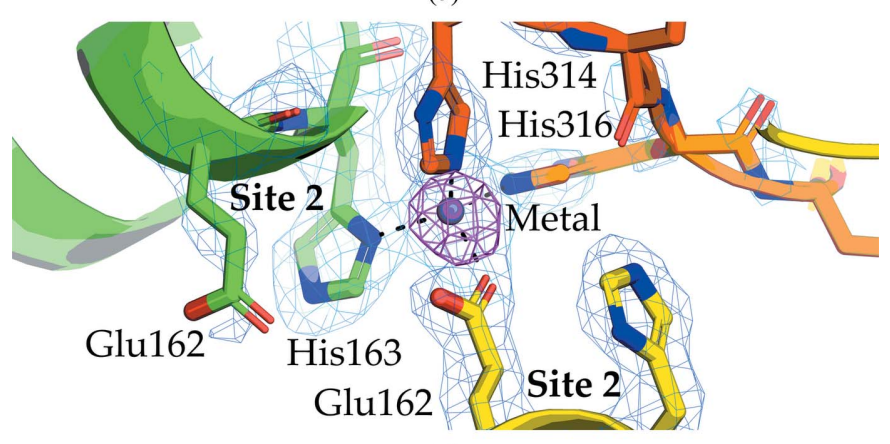

(c)

Figure 2

Enlarged views of YfeA metal-coordination complexes. Three YfeA protomers are colored green, yellow and orange. The YfeA models are overlaid with anomalous difference electron density contoured at $4 \sigma$ (magenta or orange mesh) and electron density calculated from a $2 m F_{\mathrm{o}}-D F_{\mathrm{c}}$ map contoured at $1 \sigma$ (blue mesh). (a) YfeA site 2 intercoordinated structure 1 contains square bipyramidal geometry at site 2 . (b) YfeA site 2 inter-coordinated structure 2 contains tetrahedral geometry at site 2. (c) YfeA site 2 inter-coordinated structure 3 contains tetrahedral geometry at site 2 . binding may instead come from bulk solvent and/or other surface elements.

The amino-acid sequences of YfeA homologs from 15 unique Yersinia species were compared and strains of the environmental species Y. entomophaga (GenBank accession WP_064518097.1) and Y. ruckeri (GenBank accession AJI94945.1) were found to contain a site 2 lysine substitution for glutamate or a lysine insertion between the glutamate and histidine, but the histidine is conserved in all sequences (Fig. 4). The site 2 binding capacity has not been tested for these sequences, but should these homologs bind metal at site 2 then this would indicate that site 2 does not require a paired hydrogen-bond donor or acceptor like other SBP binding sites (Ledvina et al., 1996). Instead, site 2 is in a region of negative electrostatic surface potential (Fig. 5). Binding sites with surface electrostatic potential often recruit substrates with complementary charge (Honig \& Nicholls, 1995), and the electric field can modulate and enhance or attenuate metal binding (Dudev et al., 2018). Therefore, the substrate selectivity and affinity of site 2 are likely to be influenced by favorable conditions that arise from the local polarization of an electronegative region and the availability of water molecules from bulk solvent that add to site 2 ligands to achieve tetracoordinate geometry.

Sites 1 and 2 have different local (for example electrostatic) and global (for example buried versus surface) environments that impact metal binding and possibly function. The c-clamp fold contributes all of the coordinate interactions at site 1 , limiting metal binding to a single configuration in the buried site. On the other hand, protein and bulk solvent combine to achieve all of the coordinate interactions for the site 2 surface site. Nine YfeA ancillary metal-binding sites have previously been identified (Radka et al., 2017) and three additional ancillary sites were identified in this work. These additional ancillary sites highlight multiple metal-binding sites in close proximity to site 2 that were not observed in previous work, and are jointly occupied in YfeA site 2 inter-coordinated structure 2 (Fig. 1d). This metal-binding behavior is consistent

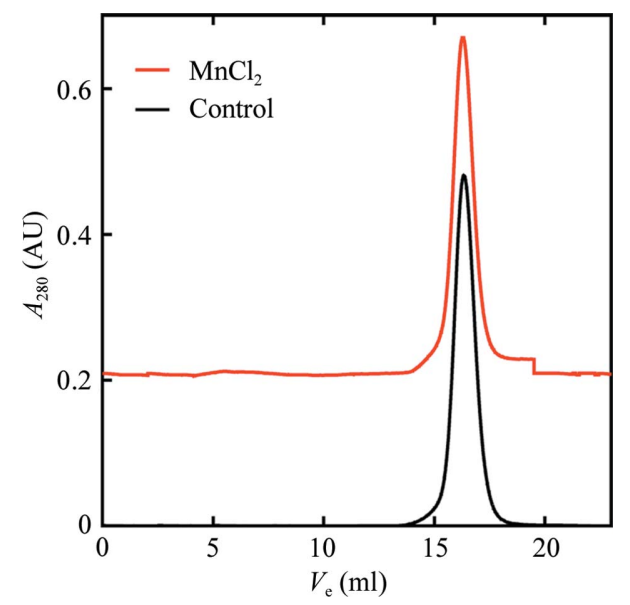

Figure 3

Gel-filtration profiles of YfeA-His 10 in the presence or absence of $\mathrm{MnCl}_{2}$. Both peaks correspond to monomeric YfeA, indicating that oligomer formation does not occur under the conditions of the experiment. 
with the characterization of YfeA as a zinc sink (Radka et al., 2017) or a metal sponge. Back-soaking experiments have demonstrated that the metals deposited across the ancillary sites are loosely bound (Radka et al., 2017) and may be scavenged by higher affinity proteins when metal abundance decreases. It is unclear whether the site 2-bound metal shares the same fate and/or purpose as the ancillary site-bound metal. Varied configurations of YfeA crystallographic packing show how multiple polypeptide chains can collaborate with site 2 to coordinate the metal. These configurations include a non-site-2 residue from the surrounding negative electrostatic region (YfeA site 2 inter-coordinated structure 1; Fig. 2a) or multiple site 2 motifs (structures 2 and 3; Figs. $2 b$ and $2 c$, respectively). Only one site 2 motif is needed to bind the metal, and the metal can be coordinated by backbone or side-chain ligands, making a potential metal handoff between proteins feasible. Participation of the $\mathrm{YfeA} \mathrm{His}{ }_{10}$ tag in metal coordination is intriguing because the zinc-binding SBP ZnuA has a histidinerich loop near ZnuA site 1 that has been implicated in metal transfer to/from site 1 (Ilari et al., 2011; Yatsunyk et al., 2008; Hecel et al., 2020). The participation of the YfeA His ${ }_{10}$ tag in collaborative metal coordination may show how a histidinerich loop in ZnuA may slip in to scavenge the metal from YfeA site 2 and deliver it to ZnuA site 1 .

Although a role for the YfeA His tag has not been seen in any of the previously published structures, the role here is somewhat artifactual but nevertheless still informative. Zinc coordination usually requires tetrahedral geometry with at least two histidine ligands. Site 2 of one YfeA molecule only contributes two of the four ligands, and only one is a histidine (His163). Therefore, His-tag zinc coordination shows that (i) the site 2 metal site is sufficiently close to the surface to participate in intermolecular metal coordination and (ii) the His-tag histidine is likely to mimic the metal coordination geometry that could occur with an as-yet-unidentified biological binding partner.

The possibility of metal transfer from YfeA site 2 to the Yfe transporter was considered and an alignment of YfeA against
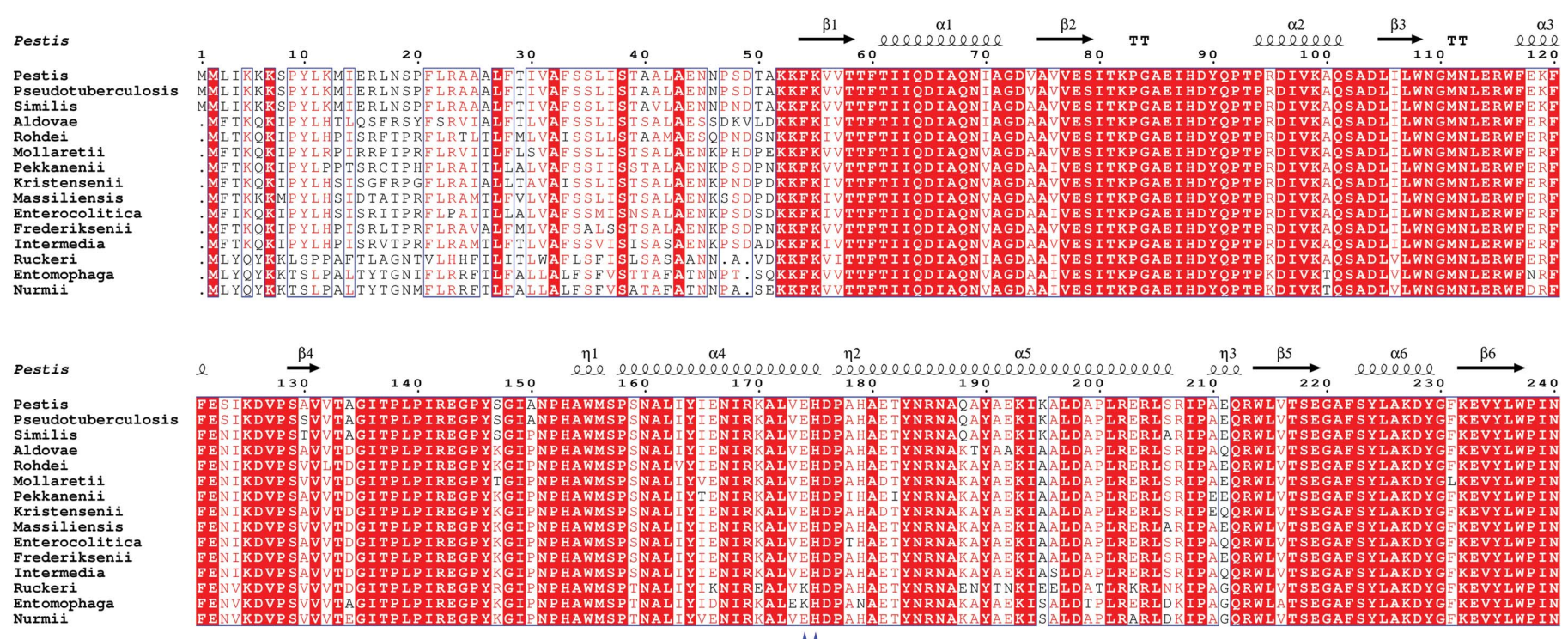

a4

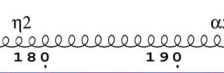

25

oceces

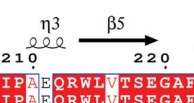
$\underset{230}{\alpha 6} \stackrel{\beta 6}{\longrightarrow}$

Pestis
Pseudotuberculosis
Similis
Aldovae
Rohdei
Mollaretii
Pekkanenii
Kristensenii
Massiliensis
Enterocolitica
Frederiksenii
Intermedia
Ruckeri
Entomophaga
Nurmii
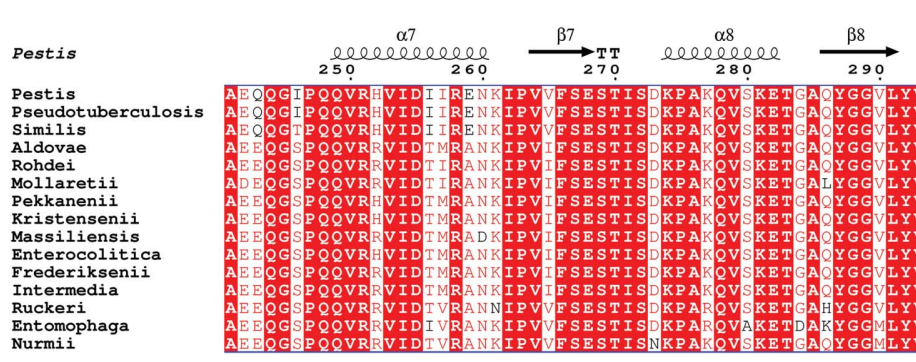

$\uparrow$ Site 2
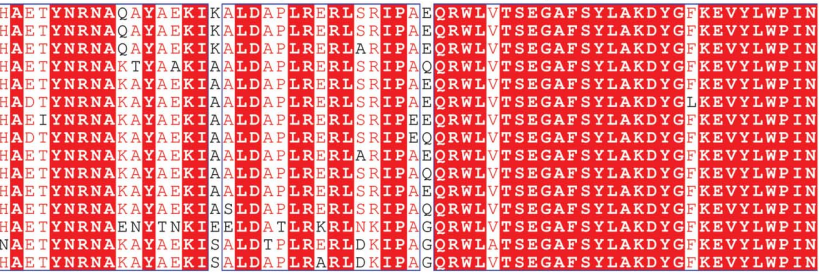

$\mathrm{TT}$
300

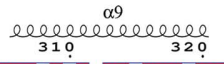

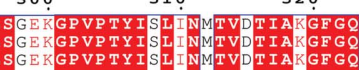

Figure 4

YfeA sequence analysis. YfeA secondary structure mapped onto a YfeA homolog sequence alignment across Yersinia species. The Y. pestis YfeA secondary-structure components (PDB entry 5uxs) are shown above a sequence alignment of Y. pestis (GenPept entry WP_002227896.1), Y. pseudotuberculosis (GenPept entry WP_072083356.1), Y. similis (GenPept entry WP_081761347.1), Y. aldovae (GenPept entry WP_049633611.1), Y. rohdei (GenPept entry WP_004713032.1), Y. mollaretii (GenPept entry WP_049677738.1), Y. pekkanenii (GenPept entry WP_049614033.1), Y. kristensenii (GenPept entry WP_050116424.1), Y. massiliensis (GenPept entry WP_019210183.1), Y. enterocolitica (GenPept entry WP_083158681.1), Y. frederiksenii (GenPept entry WP_087818068.1), Y. intermedia (GenPept entry WP_042569899.1), Y. ruckeri (GenPept entry WP_042525072.1), Y. entomophaga (GenPept entry WP_064518097.1) and Y. nurmii (GenPept entry WP_049600648.1). Site 2 amino-acid residues are identified by blue arrows under the alignment. Primary sequences of YfeA were aligned using Clustal Omega (https://www.ebi.ac.uk/Tools/msa/clustalo/) and the alignment was visualized using ESPript (http://endscript.ibcp.fr/ESPript/ESPript/index.php). Sequence numbering is that assigned to Y. pestis (GenPept entry WP_002227896.1). 


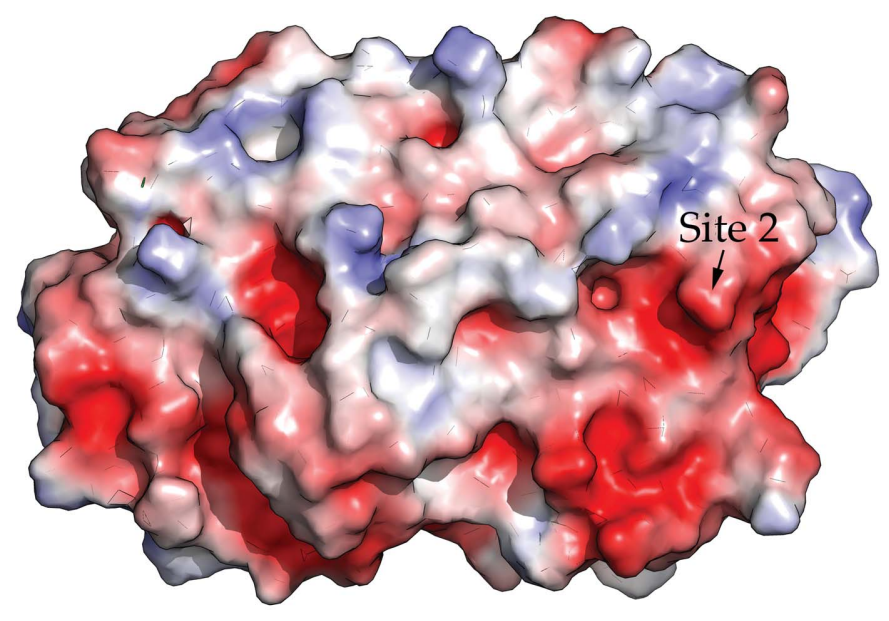

Figure 5

Site 2 is in an electronegative region of YfeA identified by a black arrow. The electrostatic surface potential of YfeA colored from red $\left(-3 k T \mathrm{e}^{-1}\right)$ to blue $\left(3 k T \mathrm{e}^{-1}\right)$ shows a distinct acidic patch surrounding site 2 .

BtuF in complex with the Btu transporter (PDB entry 4fi3; Korkhov et al., 2012) was performed. The alignment predicts that site 2 faces away from the translocation pathway of the transporter and is therefore unlikely to make meaningful contact with the YfeBCD transporter components that are equivalent to the Btu transporter components. YfeE is an additional transmembrane-protein component of the Yfe transporter for which a function has not been established and is a unique feature of the Yfe transporter (Bearden \& Perry, 1999; Bearden et al., 1998). There is no homology model for YfeE, which may also be a potential binding partner for site 2 .

\section{Acknowledgements}

Author contributions are as follows. Conceptualization, CDR and SGA; methodology, CDR and SGA; formal analysis, CDR and SGA; investigation, CDR; resources, SGA; writing original draft preparation, CDR; writing - review and editing, CDR and SGA; visualization, CDR; supervision, SGA. All authors have read and agreed to the published version of the manuscript. The data for YfeA site 2 inter-coordinated structure 1 were collected on beamline CMCF-BM at the Canadian Light Source (CLS; Fodje et al., 2014), a national research facility of the University of Saskatchewan, which is supported by the Canada Foundation for Innovation (CFI), the Natural Sciences and Engineering Research Council (NSERC), the National Research Council (NRC), the Canadian Institutes of Health Research (CIHR), the Government of Saskatchewan and the University of Saskatchewan. The date for YfeA site 2 inter-coordinated structure 2 and structure 3 data were collected on the Southeast Regional Collaborative Access Team (SER-CAT) 22-ID beamline at the Advanced Photon Source, Argonne National Laboratory (ANL). SER-CAT is supported by its member institutions and equipment grants (S10_RR25528, S10_RR028976 and S10_OD027000) from the National Institutes of Health. Use of the APS was supported by the US Department of Energy, Office of Science, Office of Basic Energy Sciences under
Contract No. W-31-109-Eng-38. The authors declare that they have no conflicts of interest with the contents of this article.

\section{Funding information}

CDR was funded by the University of Alabama at Birmingham Office of Diversity, Equity, and Inclusion.

\section{References}

Abedi, A. A., Shako, J. C., Gaudart, J., Sudre, B., Ilunga, B. K., Shamamba, S. K. B., Diatta, G., Davoust, B., Tamfum, J. M., Piarroux, R. \& Piarroux, M. (2018). Emerg. Infect. Dis. 24, 210-220.

Andrianaivoarimanana, V., Piola, P., Wagner, D. M., Rakotomanana, F., Maheriniaina, V., Andrianalimanana, S., Chanteau, S., Rahalison, L., Ratsitorahina, M. \& Rajerison, M. (2019). Emerg. Infect. Dis. 25, 220-228.

Bearden, S. W. \& Perry, R. D. (1999). Mol. Microbiol. 32, 403-414.

Bearden, S. W., Staggs, T. M. \& Perry, R. D. (1998). J. Bacteriol. 180, 1135-1147.

DeLano, W. L. (2002). PyMOL. http://www.pymol.org.

Dudev, T., Ilieva, S. \& Doudeva, L. (2018). Phys. Chem. Chem. Phys. 20, 24633-24640.

Emsley, P., Lohkamp, B., Scott, W. G. \& Cowtan, K. (2010). Acta Cryst. D66, 486-501.

Fodje, M., Grochulski, P., Janzen, K., Labiuk, S., Gorin, J. \& Berg, R. (2014). J. Synchrotron Rad. 21, 633-637.

Hecel, A., Kola, A., Valensin, D., Kozlowski, H. \& Rowinska-Zyrek, M. (2020). Inorg. Chem. 59, 1947-1958.

Honig, B. \& Nicholls, A. (1995). Science, 268, 1144-1149.

Ilari, A., Alaleona, F., Petrarca, P., Battistoni, A. \& Chiancone, E. (2011). J. Mol. Biol. 409, 630-641.

Korkhov, V. M., Mireku, S. A. \& Locher, K. P. (2012). Nature, 490, 367-372.

Laitaoja, M., Valjakka, J. \& Jänis, J. (2013). Inorg. Chem. 52, $10983-$ 10991.

Ledvina, P. S., Yao, N., Choudhary, A. \& Quiocho, F. A. (1996). Proc. Natl Acad. Sci. USA, 93, 6786-6791.

Liebschner, D., Afonine, P. V., Baker, M. L., Bunkóczi, G., Chen, V. B., Croll, T. I., Hintze, B., Hung, L.-W., Jain, S., McCoy, A. J., Moriarty, N. W., Oeffner, R. D., Poon, B. K., Prisant, M. G., Read, R. J., Richardson, J. S., Richardson, D. C., Sammito, M. D., Sobolev, O. V., Stockwell, D. H., Terwilliger, T. C., Urzhumtsev, A. G., Videau, L. L., Williams, C. J. \& Adams, P. D. (2019). Acta Cryst. D75, 861-877.

Otwinowski, Z. \& Minor, W. (1997). Methods Enzymol. 276, 307-326.

Radka, C. D., DeLucas, L. J., Wilson, L. S., Lawrenz, M. B., Perry, R. D. \& Aller, S. G. (2017). Acta Cryst. D73, 557-572.

Radka, C. D., Labiuk, S. L., DeLucas, L. J. \& Aller, S. G. (2019). Acta Cryst. D75, 831-840.

Radka, C. D., Radford, L. L., Massicano, A. V. F., DeLucas, L. J., Lapi, S. E. \& Aller, S. G. (2018). J. Vis. Exp., e57169.

Randremanana, R., Andrianaivoarimanana, V., Nikolay, B., Ramasindrazana, B., Paireau, J., Ten Bosch, Q. A., Rakotondramanga, J. M., Rahajandraibe, S., Rahelinirina, S., Rakotomanana, F., Rakotoarimanana, F. M., Randriamampionona, L. B., Razafimbia, V., De Dieu Randria, M. J., Raberahona, M., Mikaty, G., Le Guern, A. S., Rakotonjanabelo, L. A., Ndiaye, C. F., Rasolofo, V., Bertherat, E., Ratsitorahina, M., Cauchemez, S., Baril, L., Spiegel, A. \& Rajerison, M. (2019). Lancet Infect. Dis. 19, 537-545.

Respicio-Kingry, L. B., Yockey, B. M., Acayo, S., Kaggwa, J., Apangu, T., Kugeler, K. J., Eisen, R. J., Griffith, K. S., Mead, P. S., Schriefer, M. E. \& Petersen, J. M. (2016). PLoS Negl. Trop. Dis. 10, e0004360.

Scheepers, G. H., Lycklama a Nijeholt, J. A. \& Poolman, B. (2016). FEBS Lett. 590, 4393-4401.

Shi, L., Yang, G., Zhang, Z., Xia, L., Liang, Y., Tan, H., He, J., Xu, J., Song, Z., Li, W. \& Wang, P. (2018). PLoS One, 13, e0198067. 
Udayalaxmi, S., Gangula, M. R., Ravikiran, K. \& Ettaiah, P. (2020). Struct. Chem. 31, 1057-1064.

Vogler, A. J., Chan, F., Nottingham, R., Andersen, G., Drees, K., Beckstrom-Sternberg, S. M., Wagner, D. M., Chanteau, S. \& Keim, P. (2013). mBio, 4, e00623-12.

Yao, H., Jepkorir, G., Lovell, S., Nama, P. V., Weeratunga, S., Battaile, K. P. \& Rivera, M. (2011). Biochemistry, 50, 5236-5248.
Yatsunyk, L. A., Easton, J. A., Kim, L. R., Sugarbaker, S. A., Bennett, B., Breece, R. M., Vorontsov, I. I., Tierney, D. L., Crowder, M. W. \& Rosenzweig, A. C. (2008). J. Biol. Inorg. Chem. 13, 271-288.

Zwart, P. H., Afonine, P. V., Grosse-Kunstleve, R. W., Hung, L. W., Ioerger, T. R., McCoy, A. J., McKee, E., Moriarty, N. W., Read, R. J., Sacchettini, J. C., Sauter, N. K., Storoni, L. C., Terwilliger, T. C. \& Adams, P. D. (2008). Methods Mol. Biol. 426, 419-435. 